\title{
Wie relevant ist die gesetzliche Fortbildungsverpflichtung für Lehrkräfte? Eine empirische Untersuchung zur Fortbildungsteilnahme in verschiedenen deutschen Bundesländern
}

\author{
Jenny Kuschel $\mathbb{D} \cdot$ Dirk Richter $\mathbb{D} \cdot$ Rebecca Lazarides $\mathbb{D}$
}

Eingegangen: 5. November 2019 / Überarbeitet: 26. Juni 2020 / Angenommen: 18. Juli 2020 / Online publiziert: 8. September 2020

(C) Der/die Autor(en) 2020

Zusammenfassung Für Lehrkräfte besteht in Deutschland eine allgemeine Pflicht zur Fortbildung, jedoch gibt es nur in wenigen Bundesländern konkrete Vorgaben zum Umfang dieser Verpflichtung und zur Dokumentation der Fortbildungsaktivitäten. Diese Arbeit untersucht daher die Bedeutsamkeit dieser gesetzlichen Verpflichtung für die Fortbildungsteilnahme von Lehrkräften. Anhand der Daten zur Fortbildungsaktivität aus den IQB-Ländervergleichen 2011, 2012 und dem IQB-Bildungstrend 2015 wurde mittels logistischer und linearer Regressionsmodelle analysiert, inwiefern die Teilnahme an einer Fortbildung sowie die Anzahl besuchter Fortbildungen in Zusammenhang mit den in einem Bundesland bestehenden Vorgaben zum Fortbildungsumfang und der Pflicht zum Nachweis absolvierter Fortbildungen steht. Die Ergebnisse zeigen, dass die Wahrscheinlichkeit zur Teilnahme an einer Fortbildung nur in einer der drei Studien höher ausfällt, wenn eine spezifische Fortbildungspflicht besteht. Zudem lassen sich über alle betrachteten IQB-Studien hinweg positive Zusammenhänge zwischen den gesetzlichen Vorgaben zum Fortbildungsumfang und der Anzahl besuchter Fortbildungen nachweisen. Die Zugehörigkeit zu Bundesländern mit Nachweispflicht steht jedoch nicht in systematischem Zusammenhang zur Fortbildungsteilnahme. Die Ergebnisse verweisen darauf, dass sich die Teilnahme an Fortbildungen in begrenztem Ausmaß durch gesetzliche Vorgaben steuern lässt. Mögliche Implikationen für die Erhöhung der Fortbildungsteilnahme werden diskutiert.

Schlüsselwörter Fortbildungspflicht · Lehrerfortbildung · Nachweispflicht · Weiterbildung $\cdot$ Personalentwicklung

\footnotetext{
Zusatzmaterial online Zusätzliche Informationen sind in der Online-Version dieses Artikels (https:// doi.org/10.1007/s35834-020-00274-3) enthalten.
}

J. Kuschel $(\varangle) \cdot$ D. Richter $\cdot$ R. Lazarides

Universität Potsdam, Karl-Liebknecht-Straße 24-25, 14476 Potsdam, Deutschland

E-Mail: jkuschel@uni-potsdam.de 


\title{
How relevant is the legal obligation for teachers' professional development? An empirical investigation of the uptake of professional development activities in different federal states of Germany
}

\begin{abstract}
Teachers in Germany are obliged to continue their professional training throughout their career. However, only a few federal states in Germany have defined legal requirements regarding specific obligations and documentation of teachers' professional development activities. This study examines the importance of existing legal requirements for teachers' participation in professional development. Based on data of the German National Assessment Study in 2011, 2012 and 2015, we examined relationships between teachers' participation in professional development courses and two types of legal requirements: a) specification of requirement to participate in professional training; b) requirement to document completed trainings. The results of logistic and multiple linear regression show that the probability of participating in a training course is higher in only one of the three studies if there are specifications of requirements. However, results showed relations between legal specifications and the number of professional development activities. Whether federal states require the documentation of completed trainings was not systematically related to continuing education participation. Our findings indicate that participation in professional development activities can be regulated to a limited extent by legal requirements. Possible implications for increasing professional development are discussed.
\end{abstract}

Keywords Professional development · Teacher training · Legal requirements · Inservice training

\section{Einleitung}

Die Teilnahme an Fortbildungen ermöglicht Lehrkräften, ihre professionellen Kompetenzen kontinuierlich weiterzuentwickeln. Dies ist angesichts sich ständig wandelnder schulischer und gesellschaftlicher Rahmenbedingungen unabdingbar. Empirisch konnte gezeigt werden, dass der Besuch von Fortbildungen nicht nur für die Kompetenzentwicklung von Lehrkräften bedeutsam ist, sondern auch zur Verbesserung der Unterrichtsqualität von Lehrkräften beitragen kann (Garet et al. 2001; Kennedy 2016). Hohe Unterrichtsqualität ist wiederum eine wichtige Voraussetzung für das Lernen von Schülerinnen und Schülern (Darling-Hammond et al. 2009, 2017).

Ebenso besitzen Fortbildungen in den deutschen Schul- und Lehrerbildungsgesetzen sowie in den bundeslandspezifischen Verordnungen zur Lehrerbildung in Deutschland einen hohen Stellenwert und gelten als zentrale Instrumente der Unterrichts-, Personal- und Schulentwicklung (Deutscher Verein zur Förderung der Lehrerinnen- und Lehrerfortbildung 2018). In den Rechtsvorschriften der deutschen Bundesländer ist deshalb durchgängig eine allgemeine Verpflichtung zur Fortbildung für Lehrkräfte festgelegt (Avenarius und Füssel 2010). Allerdings existieren lediglich in drei Bundesländern konkrete Vorgaben zum Umfang der Fortbildungspflicht 
(Richter 2016). Darüber hinaus unterscheiden sich die rechtlichen Bestimmungen in den Bundesländern Deutschlands in der Nachweispflicht besuchter Fortbildungen (z. B. Bremer Lehrerfortbildungsverordnung 2005; Erlass der Ministerkonferenz Sachsen-Anhalts 2012).

Im Hinblick auf die Nutzung des Fortbildungsangebots zeigen Ergebnisse der regelmäßig durchgeführten Lehrkräftebefragungen des Instituts zur Qualitätsentwicklung im Bildungswesen (IQB) ${ }^{1}$, dass sich die Teilnahmequoten zwischen den Bundesländern stark unterscheiden (z. B. Hoffmann und Richter 2016). Damit stellt sich die Frage, inwiefern die Unterschiede in den Teilnahmequoten mit bundeslandspezifischen Regelungen zusammenhängen.

Bislang liegen weder nationale noch internationalen Untersuchungen dazu vor, inwiefern spezifische Verpflichtungen zur Teilnahme an Fortbildungen oder eine Verpflichtung zum Nachweis absolvierter Fortbildungen eine Bedeutung für den tatsächlichen Besuch von Fortbildungen haben.

Die Frage nach der Bedeutsamkeit von gesetzlichen Verpflichtungen ist nicht nur für das deutsche Lehrerbildungssystem relevant, sondern auch für die internationale Forschung zu Professionalisierungsprozessen bei Lehrkräften, da in der Mehrheit der OECD-Ländern die Teilnahme an Fortbildungen verpflichtend ist (OECD 2018). Hinsichtlich einer Nachweispflicht besuchter Fortbildungen liegen ebenfalls keine international vergleichbaren Daten vor. Die vorliegende Studie greift dieses Desiderat auf und untersucht, inwiefern Zusammenhänge zwischen der Fortbildungsteilnahme von Lehrkräften und der Zugehörigkeit zu Bundesländern mit und ohne konkreter Fortbildungsverpflichtung sowie zu Bundesländern mit und ohne Nachweispflicht in Deutschland bestehen. Folglich geht die Studie auch der Frage nach, inwiefern eine solche Verpflichtung ein effektives Instrument zur Steuerung der Fortbildungsteilnahme darstellt. Dabei nutzt die Studie Daten aus dem IQB-Ländervergleich 2011 und 2012 sowie dem IQB-Bildungstrend 2015 in der Bundesrepublik Deutschland.

\section{Theoretischer Hintergrund}

\subsection{Fortbildungsverpflichtung in den deutschen Bundesländern}

In allen Bundesländern der Bundesrepublik Deutschland besteht eine allgemeine Pflicht zur kontinuierlichen Fortbildung für Lehrkräfte (DVLfB 2018; Avenarius und Füssel 2010). Diese ist unter anderem in Schulgesetzen, Lehrerbildungsgesetzen oder Dienstordnungen von Lehrkräften festgelegt (Hoffmann und Richter 2016; siehe tabellarische Übersicht A1 im Anhang). Neben dieser allgemeinen Verpflichtung bestehen in einigen Bundesländern - derzeitig sind dies Hamburg, Bremen und Bayern - zusätzlich konkrete Zeitangaben zum Umfang der zu besuchenden

\footnotetext{
${ }^{1}$ Das Institut zur Qualitätsentwicklung im Bildungswesen (IQB) ist eine wissenschaftliche Einrichtung der deutschen Bundesländer für gemeinsame Aktivitäten im Bereich der Qualitätssicherung und -entwicklung im allgemeinbildenden Schulsystem. Hauptaufgabe des IQB ist die Überprüfung und Weiterentwicklung von Bildungsstandards.
} 
Tab. 1 Gesetzliche Vorgaben zur Fortbildungs- und Nachweispflicht in Hamburg, Bremen und Bayern

\begin{tabular}{lll}
\hline Bundesland & Umfang der Fortbildungspflicht & Nachweis \\
\hline Hamburg & 30h pro Jahr & Wird in Form eines Portfolios gefordert \\
Bremen & 30h pro Jahr & Wird gefordert, jedoch keine Formvorgabe \\
Bayern & 12 Tage (à 5 h zu 60 min) über 4 Jahre & Wird gefordert, jedoch keine Formvorgabe \\
\hline
\end{tabular}

Vorgaben zum Umgang der Fortbildungspflicht gelten für allgemeinbildende Schulen

Fortbildungen (Richter et al. 2012, 2013a). In der Vergangenheit gab es jedoch noch weitere Bundesländer wie beispielsweise Hessen, die konkrete Vorgaben zur Fortbildungspflicht formulierten. Die in Hamburg, Bremen und Bayern aktuell bestehenden Vorgaben zum Umfang der zu besuchenden Fortbildungen unterscheiden sich allerdings in ihrer Konkretheit (Tab. 1).

In Hamburg und in Bremen sind Lehrkräfte an allgemeinbildenden Schulen dazu verpflichtet, mindestens dreißig Fortbildungsstunden innerhalb eines Schuljahres zu besuchen, wobei nicht geleistete Stunden im folgenden Schuljahr nachgeholt werden können (Hamburger Lehrkräfte-Arbeitszeit-Verordnung 2003; Bremer Lehrerfortbildungsverordnung 2005, § 2).

Darüber hinaus sind die Lehrkräfte in Hamburg seit dem Schuljahr 2006/07 verpflichtet, ein Fortbildungsportfolio zu führen, in dem sie ihre Fortbildungsaktivitäten dokumentieren (Behörde für Schule und Berufsbildung Hamburg 2006). In Bremen besteht ebenfalls eine Pflicht zur Dokumentation der Fortbildungsaktivitäten (Bremer Lehrerfortbildungsverordnung 2005, § 5). Zudem muss in Bremen die eigene Fortbildungsplanung der Schulleitung vorlegt werden (ebd., § 4). Somit sind Lehrkräfte bereits vor dem Besuch der Fortbildungen der Schulleitung rechenschaftspflichtig und die Schulleitung kann die Wahrnehmung von Fortbildungen anordnen (ebd., $\S 9$ ). In Bayern sind Lehrkräfte laut dem Bayrischen Lehrerbildungsgesetz (1995, § 20) verpflichtet, an zwölf Fortbildungstagen (à fünf Stunden zu sechzig Minuten) über einen Zeitraum von insgesamt vier Schuljahren teilzunehmen und die Teilnahme nachzuweisen. Es ist jedoch nicht angegeben, dass der Nachweis in Form eines Portfolios o. Ä. erfolgen muss (Amtsblatt der Bayerischen Staatsministerien für Unterricht und Kultus und Wissenschaft, Forschung und Kunst 2002; DVLfB 2018).

In den übrigen Bundesländern der Bundesrepublik Deutschland bestehen auf Basis der gesetzlichen Grundlagen keine Regelungen zum Umfang der zu besuchenden Fortbildungen. Zudem gibt es in keinem Bundesland Regelungen zu den Folgen einer Nichterfüllung der Fortbildungspflicht für Lehrkräfte. Allerdings sind in einigen Bundesländern Schulleitungen berechtigt, die Wahrnehmung von Fortbildungen anzuordnen. Hierzu zählen Bremen, Hessen, Rheinland-Pfalz, Schleswig-Holstein, Sachsen-Anhalt und Thüringen. Ob und in welcher Form Schulleitungen von diesem Recht Gebrauch machen, ist jedoch nicht dokumentiert. Die Anordnung zum Besuch einer Fortbildung kann jedoch nur dann erfolgen, wenn die Schulleitung einen Überblick über die bereits absolvierten Fortbildungen hat. Dazu ist eine Dokumentation besuchter Fortbildungen von Lehrkräften notwendig. 


\subsection{Unterschiede in der Nachweispflicht zwischen den Bundesländern}

Neben der allgemeinen Fortbildungspflicht und den konkreten Vorgaben zum Umfang dieser Pflicht existieren darüber hinaus in einigen Ländern explizite Bestimmungen zum Nachweis absolvierter Fortbildungen. Obwohl in dreizehn Bundesländern keine konkreten Vorschriften zum Umfang der Fortbildungspflicht bestehen, gibt es in sechs der dreizehn Bundesländer ohne konkrete Vorschriften eine Pflicht zum Nachweis der besuchten Veranstaltungen.

$\mathrm{Zu}$ diesen Bundesländern gehören Rheinland-Pfalz, Schleswig-Holstein, Mecklenburg-Vorpommern, Sachsen-Anhalt, Thüringen und Hessen. Außerdem besteht eine Nachweispflicht in den drei Bundesländern mit konkreter Fortbildungsverpflichtung.

In welcher Form die absolvierten Fortbildungen dokumentiert werden sollen, ist unterschiedlich geregelt. Lehrkräfte in Hamburg (Hamburgisches Schulgesetz 1997, $\S 88$ ), Hessen (Hessisches Lehrerbildungsgesetz 2011, § 83), Rheinland-Pfalz (Landesgesetz zur Stärkung der inklusiven Kompetenz und der Fort- und Weiterbildung von Lehrkräften, 2015, § 10), Sachsen-Anhalt (Schulgesetz Land Sachsen-Anhalt 2018, § 30a) und Thüringen (Thüringer Lehrerbildungsgesetz 2008, § 35) müssen den Nachweis in Form eines Portfolios erbringen. Das Portfolio kann bei Mitarbeitergesprächen mit der Schulleitung herangezogen werden. Somit gibt es Länder, die zwar keine Verpflichtung zur Fortbildungsteilnahme in Form einer Mindeststundenanzahl festgelegt haben, jedoch versuchen, die Verpflichtung zur Teilnahme an Fortbildungen durch eine Rechenschaftspflicht gegenüber der Schulleitung zu erreichen.

In den vier anderen Ländern mit einer Nachweispflicht (Bremen, Bayern, Mecklenburg-Vorpommern, Schleswig-Holstein) ist gesetzlich weder vorgegeben in welcher Form die Dokumentation erfolgen soll, noch wem diese Nachweise vorzulegen sind. In den übrigen sieben Ländern besteht trotz einer allgemeinen Fortbildungspflicht keine Verpflichtung zum Nachweis an Fortbildungen - damit obliegt Lehrkräften in diesen Ländern keine Verpflichtung, das Einhalten ihrer Fortbildungspflicht nachzuweisen. Dennoch sind in einigen dieser Länder die Schulleitungen verpflichtet, die Einhaltung der Fortbildungspflicht zu überprüfen (z. B. Schulgesetz Berlin 2004, § 67). Es bleibt offen, wie die Schulleitung eine solche Überprüfung ohne einen Fortbildungsnachweis oder ein verbindliches Mitarbeitergespräch durchführen soll. Weiterhin stellt sich die Frage, ob durch die zusätzliche Verpflichtung zur Dokumentation der Fortbildungen eine indirekte Steuerung der Teilnahme erzielt werden kann.

Es liegt nahe, dass sich Lehrkräfte stärker zum Besuch von Fortbildungen verpflichtet fühlen, wenn ein Nachweis der Schulleitung vorgelegt werden muss. Somit wäre anzunehmen, dass Unterschiede in den Teilnahmequoten zwischen den Ländern mit und ohne Nachweispflicht bestehen.

Insgesamt zeigt die Auswertung der gesetzlichen Bestimmungen zur Fortbildung von Lehrkräften, dass in lediglich drei Bundesländern der Bundesrepublik Deutschland eine konkrete Fortbildungsverpflichtung besteht. In sechs weiteren Ländern besteht zumindest eine Verpflichtung zum Nachweis besuchter Fortbildungen. In den 
Tab. 2 Übersicht über gesetzliche Regelungen zur Fortbildung in den einzelnen Bundesländern

\begin{tabular}{ll}
\hline Ländergruppe & Bundesländer \\
\hline (1) konkrete Fortbildungspflicht & Hamburg $^{\mathrm{a}}$, Bremen, Bayern \\
Nachweispflicht & \\
(2) keine konkrete Fortbildungspflicht & Hessen $^{\mathrm{a}}$, Mecklenburg-Vorpommern, Sachsen-Anhalt $^{\mathrm{a}}$ \\
Nachweispflicht & Thüringen $^{\mathrm{a}}$, Schleswig-Holstein \\
(3) keine konkrete Fortbildungspflicht & Saarland, Sachsen, Niedersachsen, Nordrhein-West- \\
keine Nachweispflicht & falen, Rheinland-Pfalz ${ }^{\mathrm{b}}$, Berlin, Brandenburg, Baden- \\
& Württemberg \\
\hline
\end{tabular}

${ }^{\mathrm{a}}$ Nachweis muss in Form eines Portfolios erbracht werden

bNachweispflicht besteht erst ab 2015, weshalb es für die vorliegenden Analysen noch zur Ländergruppe 3 zugeordnet wird

sieben übrigen Ländern besteht für Lehrkräfte keine Verpflichtung zum Nachweis, inwieweit sie der allgemeinen Pflicht zur Fortbildung nachgekommen sind.

Basierend auf diesem aktuellen gesetzlichen Sachstand wurden für die vorgestellte Studie spezifische Ländergruppen gebildet (Tab. 2) und Zusammenhänge zwischen der Zugehörigkeit zu diesen Gruppen und der Fortbildungsteilnahme von Lehrkräften untersucht. Wir differenzieren zwischen folgenden drei Ländergruppen: (1) Bundesländer mit konkreter Fortbildungs- und Nachweispflicht, (2) Bundesländer ohne konkrete Fortbildungspflicht, aber mit Nachweispflicht und (3) Bundesländer ohne konkrete Fortbildungs- und Nachweispflicht.

\subsection{Empirischer Forschungsstand zur Nutzung des Fortbildungsangebots in deutschen Bundesländern}

Erkenntnisse zur bundesweiten Nutzung des Fortbildungsangebots in Deutschland legte das IQB mit den Ländervergleichen 2011 und 2012 sowie dem Bildungstrend 2015 vor. In den jeweiligen Untersuchungen wurden verschiedene Kompetenzbereiche von Schülerinnen und Schülern überprüft sowie Daten von Lehrkräften verschiedener Schulstufen erhoben. Im Rahmen des IQB-Ländervergleichs 2011 wurden Lehrkräfte der Primarstufe befragt (Stanat et al. 2012). Im IQB-Ländervergleich 2012 sowie dem IQB-Bildungstrend 2015 nahmen hingegen Lehrkräfte der Sekundarstufe I teil (Pant et al. 2013; Stanat et al. 2016). Lehrkräfte wurden in diesen drei Studien des IQB auch zu den von ihnen besuchten Fortbildungsveranstaltungen innerhalb eines Zeitraums von zwei Jahren befragt. Auf Basis dieser Daten wurden Teilnahmequoten auf Ebene der Länder bestimmt, die sich teils sehr deutlich voneinander unterscheiden.

Da für den IQB-Ländervergleich 2008/09 und den IQB-Bildungstrend 2016 keine Angaben zur Fortbildungsaktivität der Lehrkräfte erhoben wurden (Köller et al. 2011; Stanat et al. 2017) und Daten aus dem IQB-Bildungstrend 2018 zum Zeitpunkt der Erarbeitung dieses Artikels noch nicht vorlagen, beschränken sich die folgenden Ausführungen auf die IQB-Studien aus den Jahren 2011, 2012 und 2015.

Im IQB-Ländervergleich 2011 nahmen im Durchschnitt 82\% aller Lehrkräfte in den Schuljahren 2009/10 und 2010/11 an mindestens einer Fortbildung teil (Richter et al. 2012). Besonders hohe Quoten erreichten hierbei die Lehrkräfte in den Län- 
dern Thüringen und Bayern. Ebenso zeigt der Bericht, dass etwa $18 \%$ der Lehrkräfte keine Fortbildung besuchten. Dieser Anteil ist in Hamburg, einem Land mit konkreter Fortbildungspflicht, aber auch in Ländern ohne konkrete Fortbildungspflicht (Saarland und Rheinland-Pfalz) besonders hoch (Richter et al. 2012).

Für die Schuljahre 2010/11 und 2011/12 berichtet der IQB-Ländervergleich 2012 eine ähnlich hohe Teilnahmequote (Richter et al. 2013b). Besonders viele Fortbildungen besuchten Lehrkräfte in den ostdeutschen Ländern ohne konkrete Fortbildungspflicht (Thüringen, Sachsen-Anhalt und Brandenburg). Im Gegensatz zum IQB-Ländervergleich 2011 wurde auch für Hamburg, einem Land mit konkreter Fortbildungspflicht, eine hohe Teilnahmequote berichtet. Am geringsten war die Teilnahmequote in den Ländern ohne konkrete Fortbildungspflicht in RheinlandPfalz sowie Schleswig-Holstein und Nordrhein-Westfalen (Richter et al. 2013b).

Im Bildungstrend 2015 lag die Teilnahmequote an Lehrerfortbildungen bei etwa $76 \%$ und damit deutlich unter der für die Vorjahre berichteten Quote (Hoffmann und Richter 2016). Abermals nahm der größte Anteil der Lehrkräfte in Thüringen, Mecklenburg-Vorpommern sowie in Hamburg an Fortbildungen teil. Es zeigt sich somit, dass sowohl Länder mit (Hamburg) und ohne (Thüringen und Mecklenburg-Vorpommern) konkrete Fortbildungsverpflichtung eine hohe Fortbildungsteilnahmequote aufweisen. In Rheinland-Pfalz war die Teilnahmequote wiederholt mit Abstand am geringsten. Doch auch in den Ländern mit konkreter Fortbildungspflicht kamen über $20 \%$ der Lehrkräfte ihrer Fortbildungspflicht nicht nach (Hoffmann und Richter 2016).

Insgesamt zeigen die Ergebnisse, dass deutliche Unterschiede in der Teilnahme an Fortbildungen zwischen den Ländern bestehen. Es überrascht, dass es trotz einer konkreten Fortbildungsverpflichtung in bestimmten Ländern einen Anteil an Lehrkräften gibt, die nicht an Fortbildungen teilnehmen. Im Gegensatz dazu weisen Länder ohne konkrete Fortbildungsverpflichtung teilweise auch hohe Teilnahmequoten auf.

Dies sind insbesondere die ostdeutschen Länder Thüringen, Brandenburg und Sachsen-Anhalt. Zwar nimmt ein überwiegender Teil der Lehrkräfte an Fortbildungen teil, jedoch besuchen sie nur wenige Veranstaltungen mit einer geringen Stundenanzahl (Richter et al. 2013b).

\section{Fragestellungen und Hypothesen der Studie}

Die vorliegende Studie verfolgt das Ziel, Unterschiede in der Fortbildungsteilnahme von Lehrkräften in Abhängigkeit länderspezifischer Vorgaben zum verpflichtend zu besuchenden Fortbildungsumfang und zur Dokumentation der besuchten Angebote zu untersuchen.

Somit wird geprüft, inwieweit Zusammenhänge zwischen den unterschiedlichen rechtlichen Vorgaben in den Ländergruppen und der Teilnahme an Fortbildungen bestehen. Weiterhin wird geprüft, inwieweit Unterschiede zwischen den Ländergruppen in der Anzahl besuchter Fortbildungen bestehen. Ausgehend von den vorangestellten Erläuterungen lassen sich drei Fragestellungen ableiten, die mit Hilfe statistischer Verfahren überprüft werden: 
Forschungsfrage 1 Nehmen Lehrkräfte aus einem Land mit gesetzlichen Vorgaben zum Umfang der Fortbildungspflicht mit einer höheren Wahrscheinlichkeit an Fortbildungen teil als Lehrkräfte in anderen Ländern?

Aufgrund der vorangestellten Überlegungen wird angenommen, dass Lehrkräfte mit einer höheren Wahrscheinlichkeit an einer Fortbildung teilnehmen, wenn sie in einem Land arbeiten, in dem eine konkrete Fortbildungsverpflichtung besteht (Ländergruppe 1) im Vergleich zu Lehrkräften in Ländern ohne konkrete Fortbildungsverpflichtung (Ländergruppe 2 und 3 ).

Forschungsfrage 2 Nehmen Lehrkräfte aus einem Land mit einer Nachweispflicht absolvierter Fortbildungen mit einer höheren Wahrscheinlichkeit an einer Fortbildung teil als Lehrkräfte aus Ländern ohne eine konkrete Nachweispflicht?

Es wird erwartet, dass Lehrkräfte aus einem Land mit Nachweispflicht (Ländergruppe 1 und 2) mit einer höheren Wahrscheinlichkeit an einer Fortbildung teilnehmen, als jene aus einem Land ohne Nachweispflicht (Ländergruppe 3).

Forschungsfrage 3 Nehmen Lehrkräfte aus einem Land mit konkreten Vorgaben zum Umfang zu absolvierender Fortbildungen und einer Nachweispflicht an mehr Fortbildungen teil als aus Ländern ohne diese Vorgaben?

Es wird angenommen, dass Lehrkräfte an mehr Fortbildungen teilnehmen, wenn sie in einem Land mit konkreter Fortbildungspflicht (Ländergruppe 1) und Nachweispflicht (Ländergruppe 2) arbeiten im Vergleich zu Lehrkräften aus einem Land ohne diese Verpflichtungen (Ländergruppe 3). Auf Grund einer empirisch verdeutlichten Bedeutung individueller Merkmale wie dem Alter und dem Geschlecht für die Fortbildungsaktivität der Lehrkräfte, wurden diese als Kontrollvariablen in das Modell mitaufgenommen (Richter et al. 2011, 2018).

\section{Methode}

\subsection{Stichprobe}

Die durchgeführten Analysen basieren auf den Daten der IQB-Ländervergleiche aus den Jahren 2011 (Stanat et al. 2014) und 2012 (Pant et al. 2015) sowie dem IQBBildungstrend 2015 (Stanat et al. 2018). Diese drei Länderberichte wurden für die Analyse ausgewählt, da sie im Gegensatz zu den Studien des IQB aus 2008/09 und 2016 neben demografischen Angaben auch die Fortbildungsaktivitäten der jeweils befragten Lehrkräfte in allen Ländern erfassen. Ziel dieser Studien war es, die Kompetenzen von Schülerinnen und Schülern in Deutschland in den unterschiedlichen Domänen und Klassenstufen zu überprüfen.

Alle Lehrkräfte der teilnehmenden Schulklassen wurden im Rahmen einer schriftlichen Befragung gebeten, demografische Angaben zur eigenen Person zu machen und Auskunft über die berufliche Qualifikation, die Berufserfahrung sowie die Nutzung von Fort-und Weiterbildungsangeboten zu geben. Beim IQB-Ländervergleich 2011 wurden insgesamt 1816 Lehrkräfte der Primarstufe für die Fächer Deutsch und Mathematik der teilnehmenden Klassen befragt. Die Stichprobe umfasst ne- 
ben Lehrkräften an allgemeinbildenden Schulen auch Lehrkräfte an Förderschulen (Richter et al. 2012). Für die vorliegende Analyse wurden Lehrkräfte ausgeschlossen, die keine Angabe zum Besuch von Fortbildungen machten $(n=72 ; 4,0 \%)$. Die realisierte Stichprobe für diesen Zeitraum umfasst somit 1744 Lehrkräfte.

Im Rahmen des IQB-Ländervergleichs 2012 wurden Lehrkräfte der Sekundarstufe I an Regel- und Förderschulen befragt, die die Fächer Mathematik, Biologie, Chemie oder Physik bzw. den Fächerverbund Naturwissenschaften unterrichteten (Richter et al. 2013). Die realisierte Stichprobe für diese Schuljahre umfasst 4213 Lehrkräfte.

Für den IQB-Bildungstrend 2015 wurden die Angaben zur Fortbildungsaktivität von Lehrkräften für die Fächer Deutsch und Englisch in der Sekundarstufe I an allgemeinbildenden Schulen und Förderschulen erfasst (Hoffmann und Richter 2016). Insgesamt umfasste die realisierte Stichprobe 3120 Lehrkräfte.

\subsection{Instrumente}

Zur Erfassung der Fortbildungsteilnahme wurde in den Lehrkräftefragebögen für die IQB- Studien erfragt, an welchen Fortbildungen die Lehrkräfte in den letzten zwei Schuljahren teilnahmen (Richter et al. 2014; Lenski et al. 2016; Schipolowski et al. 2018). Zur Bearbeitung der Forschungsfragen 1 und 2 wurde für alle Studien eine neue Variable gebildet, die angibt, ob die entsprechende Lehrkraft in den vergangenen zwei Schuljahren an mindestens einer Fortbildungsmaßnahme teilgenommen hat (Kategorien: $1=$ ja, $0=$ nein). Zur Untersuchung der Fragestellung 3 wurde die Anzahl der besuchten Fortbildungen pro Lehrkraft als abhängige Variable verwendet.

Um die Fortbildungsteilnahme in den unterschiedlichen Ländergruppen miteinander vergleichen zu können, wurden zwei weitere dichotome Variablen erzeugt, die die Ländergruppen 1 und 2 abbilden. Die erste Ländergruppe repräsentiert die Gruppe der Länder, in der eine gesetzliche Vorgabe zum Umfang zu besuchender Fortbildungen als auch eine Nachweispflicht besteht. Die zweite Ländergruppe fasst die Länder zusammen, in denen lediglich eine Nachweispflicht vorliegt. Die dritte Ländergruppe dient als Referenzgruppe, da hier keine dieser Vorgaben existieren. Rheinland-Pfalz wird in der Analyse trotz einer aktuell bestehenden Nachweispflicht zu dieser Gruppe gezählt, da sie erst 2015 eingeführt wurde.

\subsection{Datenanalyse}

Zur Untersuchung der ersten und zweiten Fragestellung wurden logistische Regressionsmodelle mit dem Programm SPSS berechnet. Dabei dienten die untersuchten Ländergruppen 1 und 2 als unabhängige Variablen und die Fortbildungsteilnahme als abhängige Variable.

Um die Koeffizienten des Regressionsmodells beurteilen zu können, wird das Odds Ratio $(O R)$ berechnet. Dieses gibt ein Chancenverhältnis zwischen zwei Wahrscheinlichkeiten (Odds) an, indem es zwei Odds in Beziehung setzt. Es zeigt an, wie stark sich die Odds unterscheiden. Das Chancenverhältnis repräsentiert im Folgenden den jeweiligen Faktor, um den sich die Wahrscheinlichkeit für den Besuch 
einer Fortbildung verändert, wenn sich der Wert der unabhängigen Variable um eine Einheit erhöht (Kuckartz et al. 2013).

Werte des OR größer als 1 zeigen also eine erhöhte Wahrscheinlichkeit an, an einer Fortbildung teilzunehmen. Werte unter 1 repräsentieren hingegen eine verringerte Wahrscheinlichkeit, an einer Fortbildung teilzunehmen. Ein Wert von 1 bedeutet, dass die Wahrscheinlichkeit für eine Fortbildungsteilnahme als auch für keine Teilnahme gleich groß ist. Es besteht kein statistisch bedeutsamer Zusammenhang zwischen dem Prädiktor und dem Kriterium der Fortbildungsteilnahme.

Zur Prüfung der Forschungsfrage 3 wurde eine multiple lineare Regression durchgeführt. Die Ländergruppen 1 und 2 dienten erneut als unabhängige Variablen und die Anzahl besuchter Fortbildungen (kontinuierliche Variable) als abhängige Variable. Als Kontrollvariablen wurden das Alter und das Geschlecht $(0=$ männlich, $1=$ weiblich) der Lehrkräfte in die Analysen mit einbezogen, da frühere Arbeiten (Richter et al. 2011, 2018) auf die Relevanz dieser Merkmale hinwiesen.

\section{Ergebnisse}

Zunächst zeigt sich in den einzelnen Studien, dass der Anteil an Lehrkräften, die an mindestens einer Fortbildung in einem Zeitraum von zwei Jahren teilnahmen, zwischen 71,6\% und 82,2\% liegt (siehe Tab. 3). Dadurch wird deutlich, dass es in allen Datensätzen mindestens einen Anteil von ca. $17 \%$ und mehr Lehrkräften gab, die an keiner Fortbildung teilnahmen. In den einzelnen Ländergruppen liegen die Teilnahmequoten in den Ländern ohne eine konkrete Fortbildungspflicht (Ländergruppe 2 und 3) für die IQB-Ländervergleiche 2011 und 2012 über der Quote der Länder mit konkreter Fortbildungspflicht (Ländergruppe 1). In 2015 kehrt sich das Verhältnis um, sodass in Ländergruppe 1 die Teilnahmequote am höchsten ist $(91,4 \%)$.

Der Mittelwert der Anzahl besuchter Fortbildungen liegt in 2011 für Ländergruppe $1(M=3,96 ; S D=2,43)$ trotz niedrigerer Teilnahmequote über dem der Ländergruppen 2 und 3 (Ländergruppe 2: $M=3,76 ; S D=2,26$; Ländergruppe 3: $M=3,29$; $S D=2,20)$. In 2012 besuchten Lehrkräfte aus der Ländergruppe mit der höchsten Teilnahmequote (Ländergruppe 2) im Mittel die meisten Fortbildungen $(M=2,77$; $S D=2,23$ ) im Vergleich zu Lehrkräften in den anderen beiden Gruppen (Ländergruppe 1: $M=2,44 ; S D=2,31$; Ländergruppe 3: $M=2,26 ; S D=2,07)$. Für den Datensatz aus 2015 liegt der höchste Mittelwert der Anzahl besuchter Fortbildungen bei Ländergruppe $1(M=2,82 ; S D=2,26)$ im Vergleich zu den Ländergruppen $2(M=2,50$; $S D=2,20)$ und $3(M=2,09 ; S D=2,01)$.

Hinsichtlich der Anzahl absolvierter Fortbildungen pro Lehrkraft zeigen sich zwischen den einzelnen Studien Unterschiede (Abb. 1). In allen Studien nimmt ein großer Anteil der Lehrkräfte an keiner Fortbildung teil (2011: 17,5\%; 2012: 28,4\%; 2015: 29,6\%). Es gibt jedoch auch einen großen Anteil an Lehrkräften, die sechs Fortbildungen besuchen. Dieser Anteil ist in der Studie aus 2011 am größten $(31,7 \%)$.

Um zu überprüfen, inwieweit die Fortbildungsteilnahme mit einer bestimmten Ländergruppe zusammenhängt (Forschungsfrage 1 und 2), wurden logistische Re- 


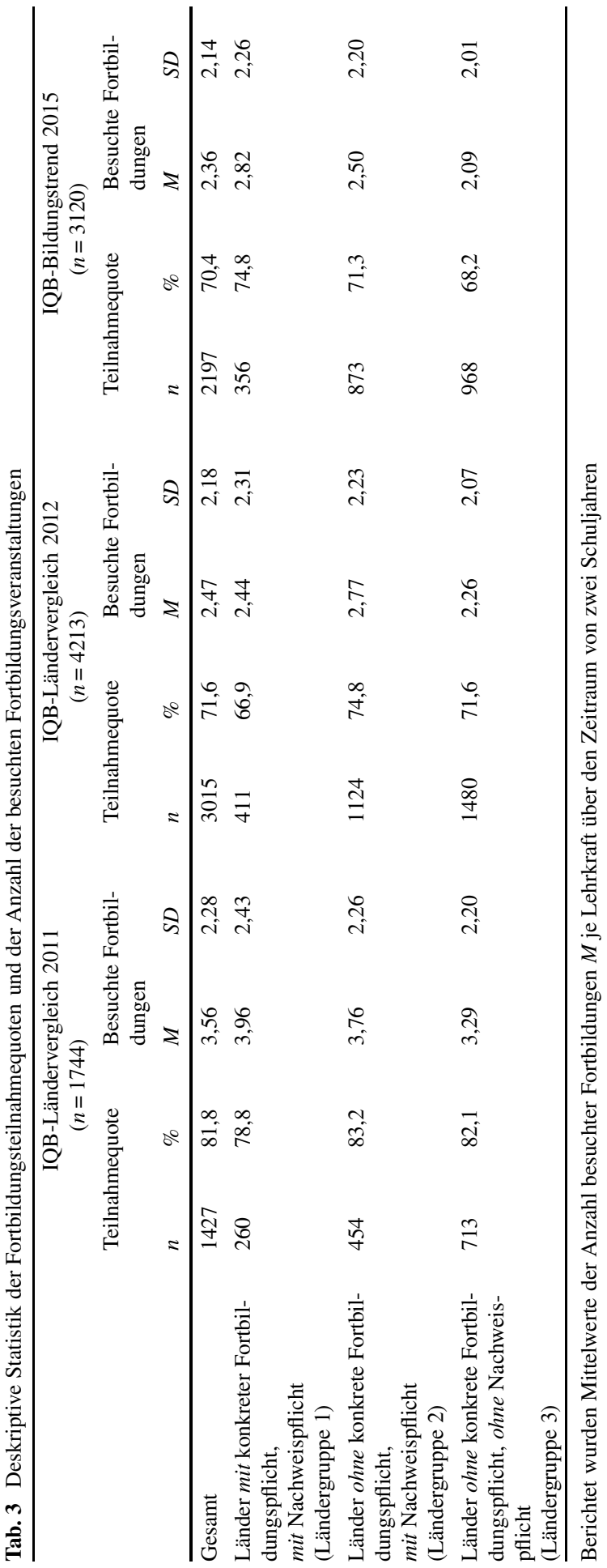




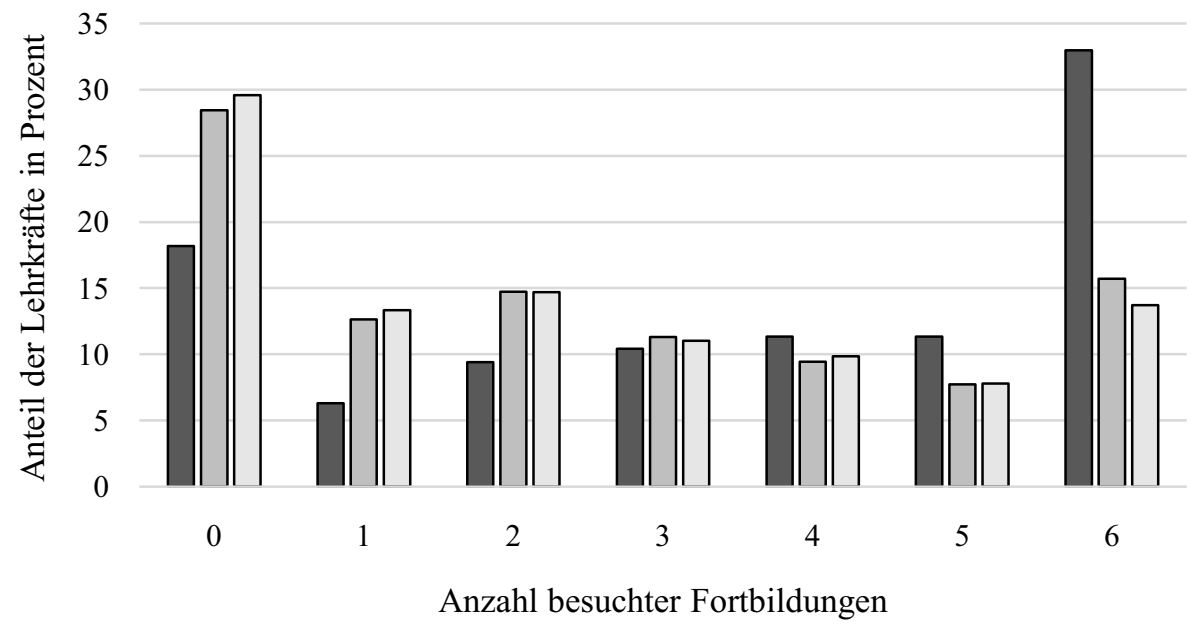

IQB-Ländervergleich 2011 口IQB-Ländervergleich 2012 口IQB-Bildungstrend 2015

Abb. 1 Prozentuale Verteilung der besuchten Fortbildungen innerhalb von zwei Schuljahren

gressionsmodelle geschätzt (Tab. 4). Zunächst zeigen sich für die Länder mit konkreter Fortbildungs- und Nachweispflicht (Ländergruppe 1) nur für die Daten aus der IQB-Studie 2015 eine höhere Wahrscheinlichkeit zur Fortbildungsteilnahme.

Lehrkräfte, die in Hamburg, Bremen oder Bayern arbeiten, nehmen mit einer fast dreimal größeren Wahrscheinlichkeit an einer Fortbildung teil als Lehrkräfte in Ländern ohne konkrete Fortbildungs- und Nachweispflicht (Ländergruppe 3). Für die Länder, in denen nur eine Nachweispflicht besteht (Ländergruppe 2), zeigen sich in keinem der Datensätze signifikante Zusammenhänge zu einer höheren Teilnahmewahrscheinlichkeit im Vergleich zur Ländergruppe 3. Hinsichtlich des Alters der Lehrkräfte zeigen sich signifikante Zusammenhänge für die Daten der IQBStudien in 2011 und 2015. Ältere Lehrkräfte nehmen mit größerer Wahrscheinlichkeit an einer Fortbildung teil als jüngere Lehrkräfte. Für das Geschlecht lassen sich ebenfalls signifikante Zusammenhänge feststellen. Frauen nehmen mit einer höheren Wahrscheinlichkeit an einer Fortbildung teil als Männer.

Zusätzlich wurde eine Analyse durchgeführt, um zu überprüfen, ob als Steuerungselement die konkrete Fortbildungspflicht oder die Nachweispflicht für die höhere Wahrscheinlichkeit einer Fortbildungsteilnahme in den jeweiligen Studien ausschlaggebend ist (siehe Tabelle A 2 im Zusatzmaterial online). In dieser Analyse wurden nicht die bereits vorgestellten Ländergruppen differenziert, sondern die beiden Merkmale (konkrete Fortbildungspflicht und Dokumentationspflicht) als unabhängige Variablen aufgeführt. Hier zeigen sich für die Daten aus dem IQB-Bildungstrend 2012 negative Zusammenhänge zwischen der konkreten Fortbildungspflicht und der Fortbildungsteilnahme. Für den Datensatz aus 2015 lassen sich hingegen positive Zusammenhänge aufzeigen. Für die Nachweispflicht sind keine signifikanten Zusammenhänge nachweisbar. 


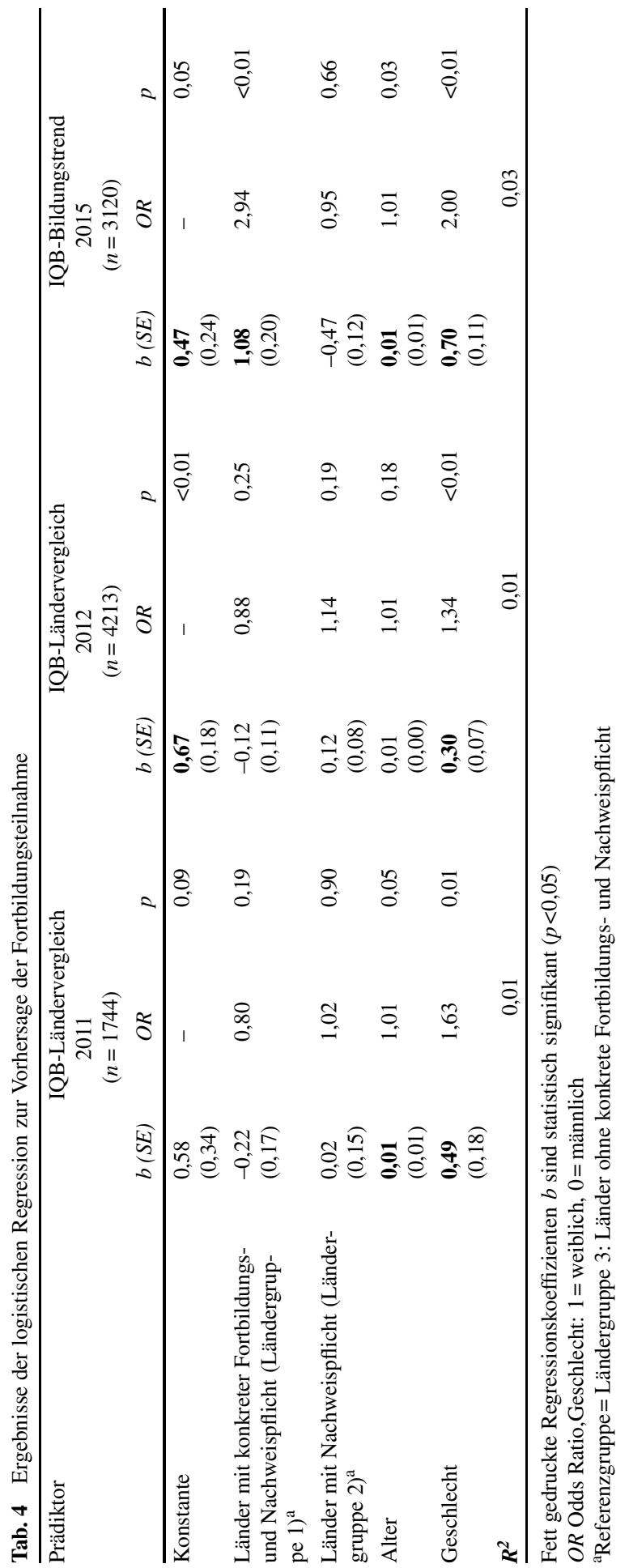




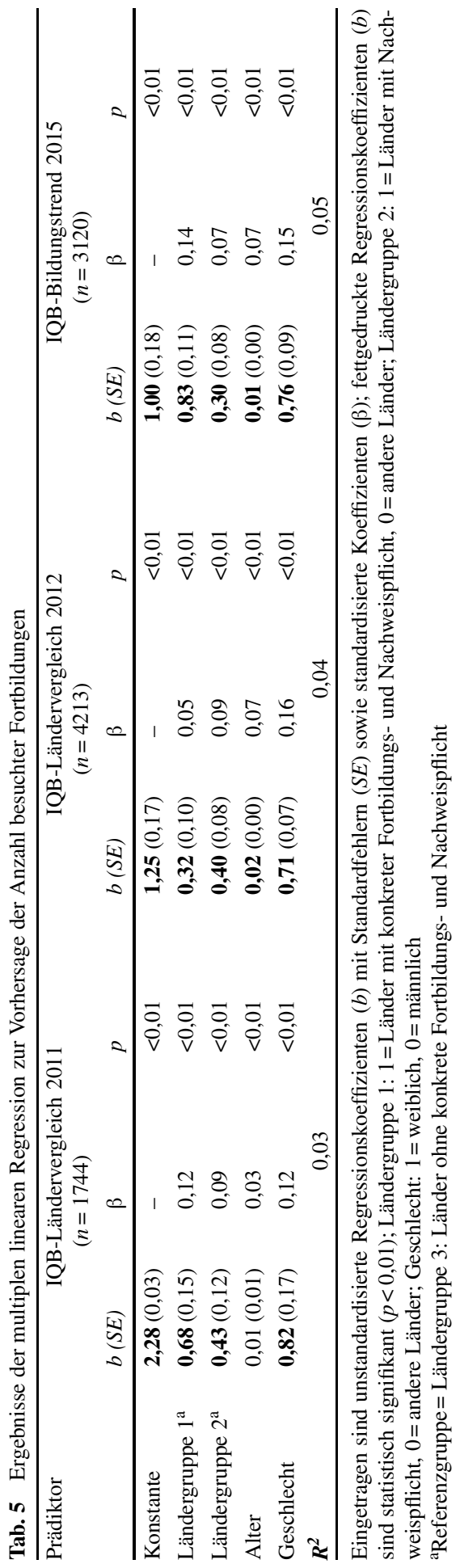


Zur Beantwortung der dritten Forschungsfrage, ob Lehrkräfte aus Ländern mit konkreter Fortbildungs-und Nachweispflicht insgesamt an mehr Fortbildungen teilnehmen, wurde eine multiple lineare Regression durchgeführt (Tab. 5). Die Ergebnisse der Analyse zeigen, dass in allen Studien im Vergleich zur Ländergruppe 3 eine höhere Wahrscheinlichkeit besteht, an mehr Fortbildungen teilzunehmen. Wenn eine konkrete Fortbildungs- und Nachweispflicht besteht (Ländergruppe 1), nehmen Lehrkräfte in diesen Ländern im Vergleich zu Lehrkräften aus Ländergruppe 3 (ohne konkrete Fortbildungs- und Nachweisplicht) an mehr Fortbildungen teil. Am stärksten ist dieser Zusammenhang für die IQB-Studie aus dem Jahr 2015. Für Lehrkräfte in den Ländern mit Nachweispflicht (Ländergruppe 2) trifft dieser Befund ebenso zu. Diese Lehrkräfte nehmen im Vergleich zu Lehrkräften der Ländergruppe 3 (ohne konkrete Fortbildungs- und Nachweisplicht) an mehr Fortbildungen teil. Der Zusammenhang ist in diesem Fall für die IQB-Studie aus 2011 am größten.

Für das Alter werden kleine Effekte für die Studien in 2012 und 2015 deutlich. Ältere Lehrkräfte nehmen an mehr Fortbildungen teil als jüngere Lehrkräfte. Bezüglich des Geschlechts der Lehrkräfte sind für alle Studien signifikante Effekte nachweisbar. Weibliche Lehrkräfte nehmen somit an mehr Fortbildungen teil als männliche Lehrkräfte.

\section{Diskussion}

Die vorliegende Studie verfolgte das Ziel, Zusammenhänge in der Teilnahme von Fortbildungen und Ländergruppen mit unterschiedlichen gesetzlichen Vorgaben in Form einer konkreten Fortbildungs- und Nachweispflicht zu analysieren und die Bedeutsamkeit dieser gesetzlichen Bestimmungen für Lehrkräfte zu überprüfen. Dazu wurden die Daten der Lehrkräftebefragung des IQB-Ländervergleichs 2011 und 2012 sowie des IQB-Bildungstrends 2015 genutzt.

Bezüglich der Forschungsfrage 1, ob Lehrkräfte, die in einem Land mit konkreter Fortbildungsverpflichtung (Ländergruppe 1) beschäftigt sind, mit höherer Wahrscheinlichkeit an einer Fortbildung teilnehmen als Lehrkräfte in anderen Ländern, lässt sich ein statistisch bedeutsamer Zusammenhang für die IQB-Studie aus 2015 nachweisen. Die erste Hypothese, dass Lehrkräfte in diesen Ländern mit einer höheren Wahrscheinlichkeit an einer Fortbildung teilnehmen, kann somit für diese Daten bestätigt werden. Für die Daten aus 2011 und 2012 trifft dies jedoch nicht zu. Insgesamt kann somit davon ausgegangen werden, dass die Verpflichtung zum Fortbildungsumfang keine konsistente Wirkung auf die Fortbildungsteilnahme hat.

Die Analysen zur Forschungsfrage 2, die sich damit befasst, inwiefern Lehrkräfte aus einem Land mit Nachweispflicht (Ländergruppe 2) mit einer höheren Wahrscheinlichkeit an einer Fortbildung teilnehmen als Lehrkräfte in einem Land ohne eine solche Verpflichtung (Ländergruppe 3), zeigen keine signifikanten Zusammenhänge. Die zweite Hypothese, dass Lehrkräfte in Ländern mit Nachweispflicht mit höherer Wahrscheinlichkeit an einer Fortbildung teilnehmen, kann deshalb nicht bestätigt werden. Die Nachweispflicht scheint keine nachweisliche Steuerungswirkung auf die Teilnahme an Fortbildungen zu haben. Dieser Befund kann möglicherweise damit erklärt werden, dass der Nutzen der Dokumentation von den Lehrkräften als zu 
gering empfunden wird und deshalb keine Steuerungswirkung entsteht. Aus einigen gesetzlichen Vorgaben der Länder geht zudem nicht klar hervor, zu welchem Zweck Fortbildungsnachweise erbracht werden sollen. Nur in fünf Ländern ist vorgesehen, dass diese zu Mitarbeitergesprächen mit der Schulleitung hinzugezogen werden. In keinem Land gibt es z.B. Regelungen für eine systematische Überprüfung der Nachweise.

In Bezug auf Forschungsfrage 3, ob Lehrkräfte aus Ländern mit konkreten Vorgaben zum Umfang zu absolvierender Fortbildungen (Ländergruppe 1) und einer bestehenden Nachweispflicht (Ländergruppe 2) an mehr Fortbildungen teilnehmen, konnte gezeigt werden, dass in diesen beiden Ländergruppen mehr Fortbildungen besucht werden als in den Ländern ohne diese Verpflichtungen (Ländergruppe 3). Die dritte Hypothese kann somit für alle Studien bestätigt werden. Eine Erklärung für die höhere Anzahl absolvierter Fortbildungen in Ländergruppe 1 könnte in der Vorgabe zum Umfang der Fortbildungspflicht liegen. Die Fortbildungspflicht in Hamburg und Bremen umfasst dreißig Stunden pro Schuljahr. Der durchschnittliche Umfang einer Fortbildungsveranstaltung umfasst in der Regel die Dauer eines Arbeitstages (Richter et al. 2010). Um die Fortbildungspflicht in Hamburg und Bremen zu erfüllen, wäre demnach die Teilnahme an mindestens drei bis vier Veranstaltungen notwendig. Die Ergebnisse lassen sich in der Art interpretieren, dass Lehrkräfte, die der Fortbildungspflicht nachkommen, dies auch im vollen Umfang tun.

Des Weiteren konnte für die Studien aus 2011 und 2015 gezeigt werden, dass ältere Lehrkräfte fortbildungsaktiver sind als jüngere Lehrkräfte. Richter et al. (2011) beschreiben in ihrer Studie einen kurvilinearen Zusammenhang zwischen der Fortbildungsaktivität und dem Alter der Lehrkräfte. In der vorliegenden Studie wurde lediglich für das Alter der Lehrkräfte kontrolliert, sodass ein direkter Vergleich mit den Ergebnissen von Richter et al. (2011), in der kurvilineare Zusammenhänge untersucht wurden, nicht möglich ist.

Hinsichtlich der Limitationen der Studie ist anzumerken, dass alle Angaben in der vorliegenden Studie auf freiwilligen, retrospektiven Selbstberichten beruhen. Verzerrungen der Angaben im Sinne sozialer Erwünschtheit (Bogner und Landrock 2015) oder durch Erinnerungsfehler (Reimer 2001) sind durchaus möglich. Weiterhin konnte die Mehrebenenstruktur der Daten bei den Analysen nicht berücksichtigt werden, da dafür nötige Länderkennungen nicht vorlagen. Dies hat zur Folge, dass die Standardfehler der Regressionskoeffizienten unterschätzt werden. Daher besteht die Gefahr, Effekte fälschlicher Weise als statistisch signifikant einzustufen, die es in der Grundgesamtheit jedoch nicht sind (Hinz 2009).

Schließlich konnte zwar ein signifikanter Zusammenhang zwischen dem Bestehen einer konkreten Fortbildungspflicht und der Teilnahme für die Zahlen aus dem Jahr 2015 gezeigt werden, dieser trägt jedoch nur bedingt zur Varianzaufklärung der Fortbildungsteilnahme bei. Folglich gibt es noch weitere Faktoren, die das Teilnahmeverhalten von Lehrkräften beeinflussen. Für weitere Studien sollten sowohl Faktoren auf individueller Ebene, wie der Lehrerfortbildungsmotivation (Rzejak et al. 2014; Richter et al. 2019), als auch auf institutioneller Ebene, wie z. B. schulischen Rahmenbedingungen (Richter et al. 2018), in den Blick genommen werden.

Ein maßgeblicher Beitrag der vorliegenden Studie zur aktuellen Bildungsforschung ist die erstmalige Untersuchung der Fortbildungsbeteiligung im Zusammen- 
hang mit länderspezifischen Vorgaben zur Fortbildungspflicht. Es konnte gezeigt werden, dass sich das Teilnahmeverhalten von Lehrkräften durch gesetzliche Verpflichtungen nur begrenzt steuern lässt. Zudem wurde in den untersuchten Datensätzen ein Anteil an Lehrkräften deutlich, der an keiner Fortbildung teilnahm. Folglich wäre es sinnvoll, über weitere Möglichkeiten zur Erhöhung der Teilnahme nachzudenken, beispielsweise die systematische Implementation effektiver Fortbildungsanreize. Solche Anreize werden auch ohne allgemeine Verpflichtung in anderen europäischen Ländern bereits umgesetzt. In der Slowakei und in Spanien beispielsweise ist die Teilnahme an Fortbildungen zwar freiwillig geregelt, jedoch mit Gehaltserhöhungen und beruflichem Aufstieg verknüpft (Hendriks und Scheerens 2010).

Insgesamt verdeutlichen die Ergebnisse dieser Studie, dass ein hoher Bedarf an weiterführenden Untersuchungen zu effektiven Steuerungsinstrumenten im Bereich der Lehrerfortbildungen in Deutschland besteht. Die Fortbildungsteilnahme fällt trotz bestehender Regelungen sehr heterogen aus. Aufgrund der Bedeutung von Fortbildungen für die Unterrichtsqualität (Garet et al. 2001; Kennedy 2016) und Lernentwicklung von Schülerinnen und Schülern (Darling-Hammond et al. 2009, 2017) sind Untersuchungen zur Nutzung von Fortbildungen von großem Interesse für die aktuelle Bildungsforschung.

Funding Open Access funding provided by Projekt DEAL.

Open Access Dieser Artikel wird unter der Creative Commons Namensnennung 4.0 International Lizenz veröffentlicht, welche die Nutzung, Vervielfältigung, Bearbeitung, Verbreitung und Wiedergabe in jeglichem Medium und Format erlaubt, sofern Sie den/die ursprünglichen Autor(en) und die Quelle ordnungsgemäß nennen, einen Link zur Creative Commons Lizenz beifügen und angeben, ob Änderungen vorgenommen wurden.

Die in diesem Artikel enthaltenen Bilder und sonstiges Drittmaterial unterliegen ebenfalls der genannten Creative Commons Lizenz, sofern sich aus der Abbildungslegende nichts anderes ergibt. Sofern das betreffende Material nicht unter der genannten Creative Commons Lizenz steht und die betreffende Handlung nicht nach gesetzlichen Vorschriften erlaubt ist, ist für die oben aufgeführten Weiterverwendungen des Materials die Einwilligung des jeweiligen Rechteinhabers einzuholen.

Weitere Details zur Lizenz entnehmen Sie bitte der Lizenzinformation auf http://creativecommons.org/ licenses/by/4.0/deed.de.

\section{Literatur}

Avenarius, H., \& Füssel, H.-P. (2010). Schulrecht. Ein Handbuch für die Praxis, Rechtsprechung und Wissenschaft. Kronach: Carl Link.

Bayerische Staatskanzlei (1995). Bayrisches Lehrerbildungsgesetz (BayLBG). München: Bayerische Staatskanzlei.

Bayerisches Staatsministeriums für Unterricht und Kultus und Wissenschaft, Forschung und Kunst (2002). Fortbildungsangebote und Fortbildungsverpflichtung. Amtsblatt Nr. 16 vom 9. August 2002. München: Bayerisches Staatsministeriums für Unterricht und Kultus und Wissenschaft, Forschung und Kunst.

Behörde für Schule und Berufsbildung (1997). Hamburgisches Schulgesetz (HMBSG). Hamburg: Behörde für Schule und Berufsbildung.

Behörde für Schule und Berufsbildung (2003). Verordnung über die Arbeitszeit der Lehrerinnen und Lehrer an staatlichen Schulen (Lehrkräfte-Arbeitszeit-Verordnung-LehrArbzVO). Hamburg: Behörde für Schule und Berufsbildung. 
Behörde für Schule und Berufsbildung (2006). Hinweise zum Fortbildungsportfolio. Hamburg: Behörde für Schule und Berufsbildung.

Bogner, K., \& Landrock, U. (2015). Antworttendenzen. GESIS Survey Guidelines. Mannheim: GESIS Leibniz-Institut für Sozialwissenschaften. https://doi.org/10.15465/gesis-sg_016.

Darling-Hammond, L., Chung Wei, R., Andree, A., Richardson, N., \& Orphanos, S. (2009). Professional learning in the learning profession: a status report on teacher development in the United States and abroad. Stanford, CA: Stanford University, National Staff Development Council.

Darling-Hammond, L., Hyler, M.E., \& Gardner, M. (2017). Effective teacher professional development. Palo Alto, CA: Learning Policy Institute.

Deutscher Verein zur Förderung der Lehrerinnen und Lehrerfortbildung e. V. (DVLfB) (Hrsg.). (2018). Recherchen für eine Bestandsaufnahme der Lehrkräftefortbildung in Deutschland. Ergebnisse des Projektes Qualitätsentwicklung in der der Lehrkräftefortbildung. Teil 1. Berlin: DVLfB.

Die Senatorin für Kinder und Bildung (2005). Verordnung über die Fortbildung der Lehrkräfte und Lehrer in besonderer Funktion an öffentlichen Schulen (Lehrerfortbildungsverordnung). Bremen: Die Senatorin für Kinder und Bildung.

Garet, M.S., Porter, A.C., Desimore, L., \& Yoon, K.S. (2001). What makes professional development effective? Results from a national sample of teachers. American Educational Research Journal, 38(4), 915-945.

Hendriks, M. A., \& Scheerens, J. (2010). Teacher' professional development in Europe: results from earlier studies. In J. Scheerens (Hrsg.), Teachers' professional development. Europe in international comparison. A secondary analysis based on the TALIS dataset (S. 43-56). Luxemburg: OECD European Union.

Hessisches Kultusministerium (2011). Hessisches Lehrerbildungsgesetz (HLbG). Wiesbaden: Hessisches Kultusministerium.

Hinz, T. (2009). Mehrebenenanalyse. In S. Kühl, P. Strodtholz \& A. Taffersthofer (Hrsg.), Handbuch Methoden der Organisationsforschung (S. 648-667). Münster: VS.

Hoffmann, L., \& Richter, D. (2016). Aspekte der Aus- und Fortbildung von Deutsch- und Englischlehrkräften im Ländervergleich. In P. Stanat, K. Böhme, S. Schipolowski \& N. Haag (Hrsg.), IQB-Bildungstrend 2015. Sprachliche Kompetenzen am Ende der 9. Jahrgangsstufe im zweiten Ländervergleich (S. 481-508). Münster, New York: Waxmann.

Kennedy, M. M. (2016). How does professional development improve teaching? Review of Educational Research, 86(4), 945-980.

Köller, O., Knigge, M. \& Tesch, B. (Hrsg.) (2010). Sprachliche Kompetenzen im Ländervergleich. Münster, New York, München, Berlin: Waxmann.

Kuckartz, U., Rädiker, S., Ebert, T., \& Schehl, J. (2013). Statistik. Eine verständliche Einführung. Wiesbaden: Springer.

Lenski, A. E., Hecht, M., Penk, C., Milles, F., Mezger, M., Heitmann, P., Stanat, P. \& Pant, H. A. (2016). IQB-Ländervergleich 2012. Skalenhandbuch zur Dokumentation der Erhebungsinstrumente. Berlin: Humboldt-Universität zu Berlin, Institut zur Qualitätsentwicklung im Bildungswesen. https://doi.org/ $10.18452 / 3125$

Ministerium für Bildung des Landes Sachsen-Anhalt (2012). Erlass der Ministerkonferenz vom 19.11.2012: Schule als professionelle Lerngemeinschaft. Magdeburg: Ministerium für Bildung des Landes Sachsen-Anhalt.

Ministerium für Bildung (2015). Landesgesetz zur Stärkung der inklusiven Kompetenz und der Fort- und Weiterbildung von Lehrkräften (IKFWBLehrG). Mainz.

Ministerium für Bildung des Landes Sachsen-Anhalt (2018). Schulgesetz des Landes Sachsen-Anhalt (SchulG LSA). Magdeburg: Ministerium für Bildung des Landes Sachsen-Anhalt.

OECD (2018). Bildung auf einen Blick 2018. OECD-Indikatoren. Paris: OECD.

Pant, H. A., Stanat, P., Schroeders, U., Roppelt, A., Siegle, T., \& Pöhlmann, C. (2013). Ländervergleich 2012. Mathematische und Naturwissenschaftliche Kompetenzen am Ende der Sekundarstufe I. Münster, New York, München, Berlin: Waxmann.

Pant, H. A., Stanat, P., Hecht, M., Heitmann, P., Jansen, M., Lenski, A. E., Penk, C., Pohlmann, C., Roppelt, A., Schröders, U., Siegele, T., \& IQB - Institut zur Qualitätsentwicklung im Bildungswesen (2012). IQB-Ländervergleich Mathematik und Naturwissenschaften 2012 (IQB-LV 2012). Version 3, https:// doi.org/10.5159/IQB_LV_2012_v3.

Reimer, M. (2001). Die Zuverlässigkeit des autobiographischen Gedächtnisses und die Validität retrospektiverhobener Lebensverlaufsdaten. Kognitive und erhebungspragmatische Aspekte. Berlin: MaxPlanck-Institut für Bildungsforschung. 
Richter, D. (2016). Lehrerinnen und Lehrer lernen: Fort- und Weiterbildung im Lehrerberuf. In M. Rothland (Hrsg.), Beruf Lehrer/Lehrerin: Ein Studienbuch (S. 245-260). Stuttgart: UTB.

Richter, D., Kunter, M., Anders, Y., Klusmann, U., Lüdtke, O., \& Baumert, J. (2010). Inhalte und Prädiktoren beruflicher Fortbildung von Mathematiklehrkräften. Empirische Pädagogik, 24(2), 151-168.

Richter, D., Kunter, M., Klusmann, U., Lüdtke, O., \& Baumert, J. (2011). Professional development across the teaching career. Teachers' uptake of formal and informal learning opportunities. Teaching and Teacher Education, 27(1), 116-126.

Richter, D., Kuhl, P., Reimers, H., \& Pant, H. A. (2012). Aspekte der Aus- und Fortbildung von Lehrkräften in der Primarstufe. In P. Stanat, H. A. Pant, K. Böhme \& D. Richter (Hrsg.), Kompetenzen von Schülerinnen und Schülern am Ende der vierten Jahrgangsstufe in den Fächern Deutsch und Mathematik. Ergebnisse des IQB-Ländervergleichs 2011 (S. 237-250). Münster, New York, München, Berlin: Waxmann.

Richter, D., Engelbert, M., Weirich, S., \& Pant, H. A. (2013a). Differentielle Teilnahme an Lehrerfortbildung und deren Zusammenhang zu professionsbezogenen Merkmalen von Lehrkräften. Zeitschrift für Pädagogische Psychologie, 27(3), 193-207. https://doi.org/10.1024/1010-0652/a000104.

Richter, D., Kuhl, P., Haag, N., \& Pant, H. A. (2013b). Aspekte der Aus- und Fortbildung von Mathematik und Naturwissenschaftslehrkräften im Ländervergleich. In H. A. Pant, P. Stanat, U. Schroeders, A. Roppelt, T. Siegle \& C. Pöhlmann (Hrsg.), IQB-Ländervergleich 2012. Mathematische und naturwissenschaftliche Kompetenzen am Ende der Sekundarstufe I (S. 367-390). Münster: Waxmann.

Richter, D., Böhme, K., Bastian-Wurzel, J., Pant, H. A. \& Stanat, P. (2014). IQB-Ländervergleich 2011. Skalenhandbuch zur Dokumentation der Erhebungsinstrumente. Berlin: Humboldt-Universität zu Berlin, Institut zur Qualitätsentwicklung im Bildungswesen.

Richter, E., Richter, D., \& Marx, A. (2018). Was hindert Lehrkräfte an Fortbildungen teilzunehmen. Eine empirische Untersuchung der Teilnahmebarrieren von Lehrkräften der Sekundarstufe I in Deutschland. Zeitschrift für Erziehungswissenschaft, 21(5), 1021-1043. https://doi.org/10.1007/s11618-0180820-4.

Richter, D., Kleinknecht, M., \& Gröschner, A. (2019). What motivates teachers to participate in professional development? An empirical investigation of motivational orientations and the uptake of formal learning opportunities. Teaching and Teacher Education, 86, 102929. https://doi.org/10.1016/j.tate. 2019.102929.

Rzejak, D., Künsting, J., Lipowsky, F., Fischer, E., Dezhgahi, U., \& Reichardt, A. (2014). Facetten der Lehrerfortbildungsmotivation - eine faktorenanalytische Betrachtung. Journal for Educational Research online, 6(1), 139-159.

Schipolowski, S., Haag, N., Milles, F., Pietz, S. \& Stanat, P. (2018). IQB-Bildungstrend 2015. Skalenhandbuch zur Dokumentation der Erhebungsinstrumente in den Fächern Deutsch und Englisch. Berlin: Humboldt-Universität zu Berlin, Institut zur Qualitätsentwicklung im Bildungswesen.

Senatsverwaltung für Bildung, Jugend und Familie (2004). Schulgesetz für das Land Berlin (SchulG). Berlin: Senatsverwaltung für Bildung, Jugend und Familie.

Stanat, P., Pant, H. A., Böhme, K., \& Richter, D. (Hrsg.). (2012). Kompetenzen von Schülerinnen und Schülern am Ende der vierten Jahrgangsstufe in den Fächern Deutsch und Mathematik. Ergebnisse des IQB-Ländervergleichs 2011. Münster, New York, München, Berlin: Waxmann.

Stanat, P., Pant, H. A., Böhme, K., Richter, D., Weirich, S., Haag, N., Roppelt, A., Engelbert, M., \& Reimers, H. (2014). IQB-Ländervergleich Primarstufe 2011 (IQB-LV 2011), Version 2, https://doi. org/10.5159/IQB_LV_2011_v2.

Stanat, P., Böhme, K., Schipolowski, S., \& Haag, N. (2016). IQB-Bildungstrend 2015. Sprachliche Kompetenzen am Ende der 9. Jahrgangsstufe im zweiten Ländervergleich. Münster, New York: Waxmann.

Stanat, P., Schipolowski, S., Rojosk, C., Weirich, S., \& Haag, N. (2017). IQB-Bildungstrend 2016. Kompetenzen in den Fächern Deutsch und Mathematik am Ende der 4. Jahrgangsstufe im zweiten Ländervergleich. Münster, New York: Waxmann.

Stanat, P., Böhme, K., Schipolowski, S., Haag, N., Weirich, S., Sachse, K.A., Hoffmann, L., \& Federlein, F. (2018). IQB-Bildungstrend Sprachen 2015 (IQB-BT 2015), Version 2, https://doi.org/10.5159/IQB_ BT_2015_v2.

Thüringer Ministerium für Bildung, Jugend und Sport (2008). Thüringer Schulgesetz (ThürSchulG). Erfurt: Thüringer Ministerium für Bildung, Jugend und Sport. 